\title{
Monitoring System of Mechanical Activity of the Heart and Goelocalization of the Patient Based on the Mqtt Cloud
}

\author{
${ }^{1}$ André R. Tchamda, ${ }^{2}$ R. Tchitnga, ${ }^{3}$ Francois. B. Pelap, and ${ }^{4} \mathbf{M}$. Kom \\ 1, 2 Physics Department electronic option, University of Dschang, Dschang, Cameroon; \\ ${ }^{3}$ Physics Department mechanical option, University of Dschang, Dschang , Cameroon; \\ ${ }^{4}$ Department of Electrical Engineering and Telecommunication, University of Yaounde I, Cameroon \\ andre.tchamda@eaphysud.org; robert.tchitnga@eaphysud.org; fbpelap@yahoo.fr; \\ kommart@yahoo.com
}

\begin{abstract}
The queue of patients for consultation and appointments that we observe in our health centers is growing in size from day to day given the exponential growth of the human population. Medical systems based on telemonitoring have been developed to remotely monitor the health status of patients. In this work, we constructed a medical prototype for remote monitoring of the mechanical activity of the heart and for geolacalization of the patient based on Message Queue Telemetry Transport (MQTT). The prototype will be used by individuals or by health centers without a doctor. The geolacalization will enable us to locate the injured individual. The physiological data and GPS coordinates are collected using a portable kit and are transmitted directly to an MQTT cloud using a Wi-Fi hotspot. A web server hosted in the kit allows the user to enter the service set identifier (SSID) of the access point through a smartphone. MQTT cloud will take care of the storage, geolacalization and transmission of the signal from the mechanical activity of the heart to the doctors. Almost all smart terminals with a web browser can easily access data (smartphone, tablet, computer). Tests carried out on individuals reveal that the proposed prototype is reliable in sampling, real-time transmission and geolacalization in street map and imagery, which can help in diagnosis and rapid intervention.
\end{abstract}

Keywords: heart activity; telemonitoring; MQTT Cloud; internet; geolacalization.

\section{Introduction}

OASIS, (Organization for the Advancement of Structured Information Standards), a global consortium working on the standardization of open file formats based on XML, has just announced that it has chosen the Message Queuing Telemetry Transport (MQTT) as the message protocol for Internet of the Objects, opening the doors to a market of several million dollars. "Today, one of the big challenges is that there is no clear open standard to enable embedded systems to communicate with each other," said Mike Riegel, vice president of marketing, Mobile Solutions Group IBM. Historically, we know that if we do not have an open standard like this, it is not possible to make the necessary progress", he added. "Just as the HTTP standard has paved the way for information exchange on the web, the MQTT could set the stage for bringing billions of low-cost telemetry terminals online to collect embedded data," Said the 
vice president of IBM [1]. The GSM Association (GSMA) estimates that by 2020, 15 billion terminals of this type will be put online. And Cisco believes that this market could represent more than $\$ 14$ billion in sales for IT providers.

MQTT is an open protocol, simple, lightweight and easy to implement. This protocol is ideal to respond to the following needs: Particularly adapted to use a very low bandwidth, Ideal for use on wireless networks, Low power consumption, Very fast, it allows a response time superior to other present standards of the web. Provides high reliability if needed, requires few processor and memory resources. MQTT is a simple and lightweight TCP / IP messaging service. Messages are sent by publishers on a Topic to a broker. These messages can be read by subscribers. Topics can have a hierarchy that allows you to fine-tune the information you want. In the MQTT protocol, exchanges are secured at several levels: Transport and Authentication.

MQTT is a publication/subscription (Pub / Sub) messaging protocol that is particularly well suited to work in an environment with limited computing power and minimal network connectivity. IBM and the supplier of Eurotech systems developed the MQTT before contributing to Oasis. The protocol is already used in a wide variety of embedded systems. For example, oil and gas companies use the MQTT to monitor thousands of kilometers of pipelines. Large livestock farms, large farm structures use this protocol. Today, almost all the physical objects we see (appliances, appliances, etc.) are also connected to the MQTT. To strengthen the MQTT, Oasis has set up a new technical committee. Its objective: to ensure that the MQTT is able to work with other types of networks, devices and software applications. The engineers from Cisco, IBM, Red Hat, Software AG and Tibco, and others will support the project.

The financing of medical care has become the most important point these days and the most costly for governments. Health and medical care can also benefit fully from the deployment of MQTTs for remote diagnosis, remote health surveillance and care for the elderly [2]. MQTT should reduce patients' consultation and transportation costs. It will also bring physicians closer to some patients who live in the isolated areas without health centers. This MQTT protocol can also be used to geolocate patients requiring rapid intervention and are not able to issue an alarm signal. This is one of the points we have developed in this work. In this work, we constructed a prototype based on MQTT to monitor the mechanical activity of the heart. The prototype can also take into account the measurement of the electrical activity of the heart by just replacing the sensor module. The prototype is equipped with a microcontroller integrated circuit with Wi-Fi connection called EPS-12E. This circuit will first connect to a Wi-Fi access point, secondly will take care of recovering signals and transmitting them on the MQTT canvas for viewing by the doctor. The integrated circuit will also retrieve the geolocalization coordinates and transmit it on the MQTT canvas for a patient localization. This prototype with a powerful $80 \mathrm{MHz}$ processor has the advantage of being portable and equipped with a web server hosted in the EPS-12E circuit which permits entering of the identifier of an access point, this enables the use of the module anywhere in the world as long as Internet connection is available. The web server integrated in the circuit offers a possibility of visualization of the collected signals, which will make it possible to use the prototype within a radius of $0-200 \mathrm{~m}$ without needing to connect to the internet. The data is stored in the Cloud MQTT server and can be exported as CSV file, allowing researchers and students to access the signal for application of the course on signal processing. 
André R. Tchamda, R. Tchitnga, F.B. Pelap, and M. Kom; Monitoring System of Mechanical Activity of the Heart and Goelocalization of the Patient Based on the Mqtt Cloud. Journal of Biomedical Engineering and Medical Imaging, Volume 4, No 5, Oct (2017), pp 1-8

Therefore, the system proposed in this work reveals a unique design and management compared to previous works [3-10], including the use of ESP-12E as a Web server for viewing data in a locality where an internet connection does not exist in the distance between patient and doctor. Some existing devices are limited in transmission distance, which prevents the patient from performing activities around the world [4, 11-12]. In addition, medical devices are generally very expensive devices for personal use, patients must regularly visit health centers, which will inevitably increase the burden on these centers. In order to overcome the problems illustrated above, we have built a low-cost, short-range, high-cost portable prototype with geolocalization that can be connected to all smart terminals with a web browser.

The rest of the work will be presented as follows. The section "Architecture of the monitoring system mechanical activity based on the MQTT", followed by the section "Prototype of acquisition of the mechanical activity of the heart", followed by the section "Experimental results" which we perform on a volunteer in Good health to verify the reliability of the proposed prototype, and finally the conclusion section.

\section{Architecture of Monitoring System Mechanical Activity Based on MQTT}

This architecture presented in figure 1, which mainly consists of three parts as follows: device for detecting the mechanical activity of the heart, MQTT cloud and the doctor's interface.

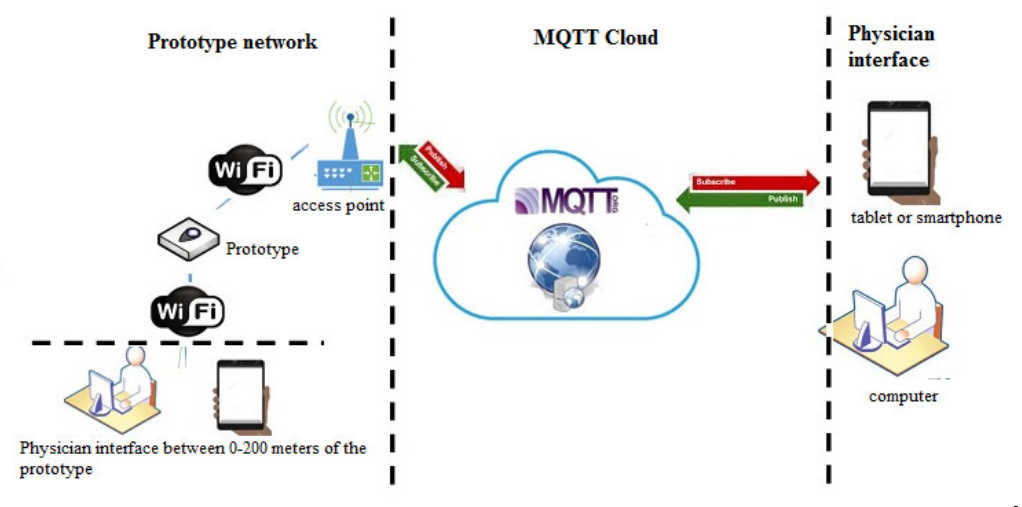

Figure 1: Architecture of the monitoring system.

\subsection{Device for Detecting the Mechanical Activity of the Heart}

This device is the departure of the entire prototype, which is responsible for the collection and transmission of physiological signals from the patient to the MQTT Cloud via a wireless Wi-Fi link. The signals will be recovered by a sensor, and will undergo amplification, filtering and conditioning before being processed by the microcontroller. The data collected by the sensor are transmitted to the MQTT cloud via the wireless Wi-Fi protocol. A GPS module is used to have the patient's coordinates, which will help to locate him for an intervention in case of danger. This protocol provides a sufficient data transfer rate for the transmission of our physiological data.

A web server is hosted in this device and plays two roles:

- First role: An SSID (Service Set Identifier) is a 32-character alphanumeric key that uniquely identifies a wireless network. Its purpose is to prevent other wireless equipment from accessing your local network, either accidentally or intentionally. The local network also has a password. 
In order for the device to connect to any access point, a web interface is provided to enter the SSID and the password of this access point.

- Second role: if the patient is close to the doctor, say a radius of 0-200 meters, there is no need using the internet for data transfer. Since the patient is close, another web interface housed in the device makes it possible to visualize the patient's signal. A signal taken from a normal person over two meters is shown in figure 5.

A smartphone, a tablet or a computer can be used to connect directly to the microcontroller's Wi-Fi (ESP-12E) to perform the first and second roles.

\subsection{MQTT Cloud}

This central part of our architecture for monitoring the mechanical activity of the heart. The MQTT cloud is used retrieve the data collected from the patient and to transmit the data to the doctor's interface. MQTT cloud consists of a data storage module, which is very important for subsequent analysis of the signal. It will also be able to export patients' data as a file (XML, JSON, CSV), which may help students and researchers during the application of signal processing. After having retrieved the coordinates coming from the GPS module, the MQTT Cloud will take care of making a geolacalization in street map and satellite imagery.

\subsection{Doctor's Interface}

This interface is accessible from all platforms using an operation system and a browser, for example: smartphone, tablet and computer. The interface provides easy access to data in the MQTT Cloud. The doctor logs in to the cloud to export the data, view the data and the patient's geographic location. The geographical location is visible on the doctor's interface in street map and satellite imagery mode, this will allow the doctor to immediately follow the patient's current location and send him an ambulance. The use of tablets and smartphones allows a great mobility of the doctor.

\section{Prototype for Acquisition of Mechanical Activity of the Heart}

The design of our prototype is presented in figure 2 and consists of: Sensor module, Controller / Wi-Fi module, GPS module, Power module.

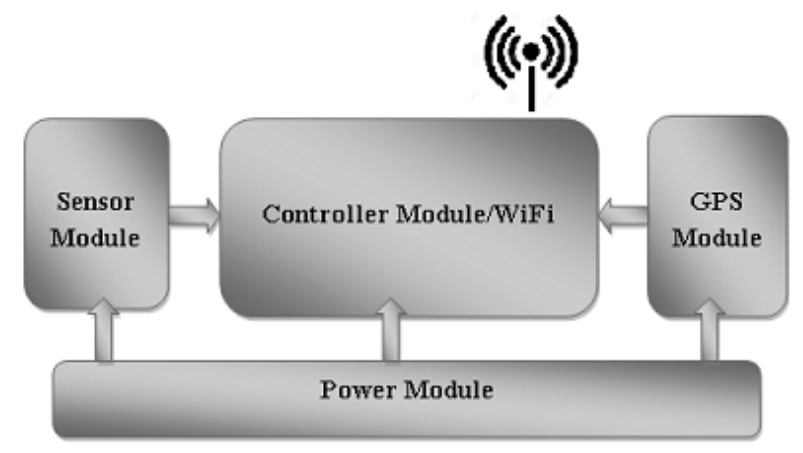

Figure 2: Components of the prototype

The architecture proposed by our prototype is less cumbersome and less expensive compared to the existing ones. Existing prototypes need an additional Bluetooth module to connect to a smartphone that transfers data to the cloud [12-13]. Other existing prototypes do not allow a geolocalization of the patient [12-15]. Figure 3 shows the physical prototype. It will be detailed as follows. 


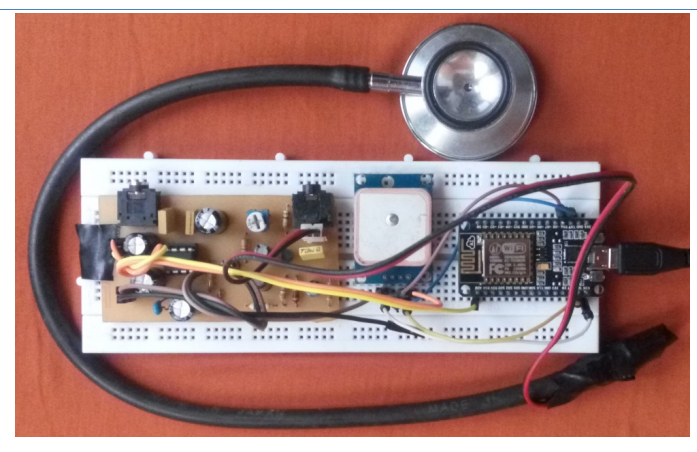

Figure 3: Physical prototype

\subsection{Sensor Module}

The module includes a Panasonic's WM-63PRT microphone used to pick up the sound produced by the mechanical movement of the heart. This microphone has an excellent frequency response with amplitude of $10-20 \mathrm{mV}$ and a frequency of $20 \mathrm{~Hz}$ to $16 \mathrm{kHz}$ [16]. The module consists of an amplifier to amplify the microphone signal followed by a Band-pass filter of $10-500 \mathrm{~Hz}$. A Band-pass filter is used to eliminate noise outside this frequency band. Finally, using the controller module, signals are collected

\subsection{Controller / Wi-Fi Module}

Controller / WI-FI module used here is ESP-12E. The ESP-12E is a low-cost Wi-Fi chip with full TCP / IP stack and MCU (microcontroller unit) capability. It is used to recover the signal at the sensor module output and send it to its built-in Wi-Fi module. This MCU is widely used in web applications, because of its Wi-Fi that supports Wi-Fi $(802.11 \mathrm{~b} / \mathrm{g} / \mathrm{n})$. The microcontroller includes 32-bit RISC CPU running at 80 $\mathrm{MHz}$, a flash memory up to 4 Mbyte and 96 Kbyte of RAM. The MCU also provides other functions such as analog to digital converter (ADC), Pulse Width Modulation (PWM), Serial Peripheral Interface Bus (SPI) and universal asynchronous receiver / transmitter (UART).

The microcontroller will sample the analog signal at the output of the sensor module. According to Shannon's theorem, the sampling frequency is set to $1 \mathrm{KHz}$, given that the measured signal is generally between $10 \mathrm{~Hz}$ and $500 \mathrm{~Hz}$.

The higher the sampling frequency, the better the reproduction of the start signal, which will cause no problem if the sensor module is replaced by another module used to measure another physiological signal and as long as the maximum value of the this signal will be less than $500 \mathrm{HZ}$. In order to respect this sampling time, we have fixed a TIMER interrupt which will take place every $1 / 1000$ seconds. The scanned data is transmitted internally to the Wi-Fi module integrated in the MCU and then routed to the Wi-Fi access point.

\subsection{GPS Module}

The GPS module used here is GY-NEO6MV2. Printed circuit boards with the indication GY-NEO6MV2 are very popular and cheap on the internet. NMEA messages are available, with a standard speed of 9600 bits / sec, at the TX output of the printed circuit board. To communicate with microcontrollers, it has a UART port. Queried by the MCU, it returns the longitude, latitude and altitude of the patient's position. This information will be sent to the MQTT broker. 


\subsection{Power Module}

The power module provides a reliable power supply to each module of our device. The power supply is via the USB port or a lithium battery.

\section{Where to Capture the Sounds Produced by the Mechanism of the Heart}

The words reserved for this are cardiac auscultation. The noises of the heart beats are mainly noises of fluids (blood) and materials (valves), more precisely: dynamic events, Contraction and relaxation of the atria and ventricles, the movement of valves. The blood flow, the turbulence of the blood at the time of closing of the valves.

The auscultation of the heart is mainly concerned with listening to these noises that occur inside the body via a stethoscope. The optimal auscultation sites (figure 4) are:

- Mitral focus: The blood passing through the mitral muscle is directed towards the tip of the heart. The best perception is at the level of the 5th intercostal space, on the mid-clavicular line, in sub-mammary position.

- Tricuspid focus: as the heart is lying at $45^{\circ}$, the blood flow through the tricuspid valve goes downwards: the tricuspid is best heard at the xiphoid focus.

- Aortic focus: the inner end of the second right intercostal space, corresponding to the main auscultation focus of the aortic sigmoid.

- Pulmonary focus: the inner end of the second left intercostal space, along the sternum, corresponding to the pulmonary sigmoid valves, incidentally to the aortic valves (accessory aortic focus extending along the left margin of the sternum).

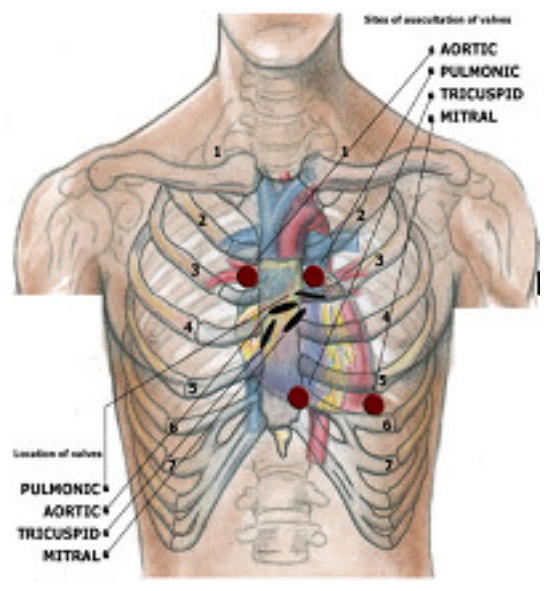

Figure 4: Cardiac Auscultation Foyers

\section{Display of Experimental Signals}

To verify the reliability of our prototyping, experiments were conducted on normal people. The prototype offers an option to view a patient's data without having to go through the cloud MQTT. This is useful when the doctor is close to the patient. An HTTP server hosted in the device can display the patient's signal on a browser. From JavaScript, the canvas element was used to create the signal. Figure 5 shows the display of the signal over a very short distance between doctor and patient 


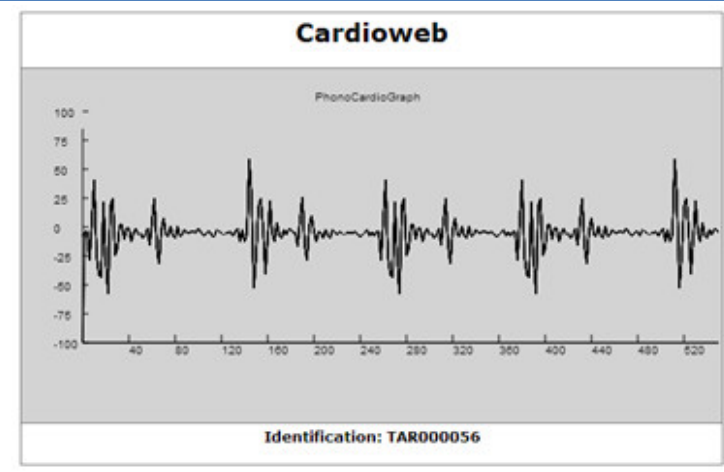

Figure 5: Example of visualization by integrated web server

Figure 6 shows the reception of the data via the MQTT. It shows us the reception of a signal describing the mechanical activity of the heart. We will also notice two images to the right of the curve. These images are images showing the geolocalization of the patient in two modes (street map, satellite imagery). The blue dot on these images marks the position of the patient. An error margin with respect to this point of 8.5 meters must be taken into account.

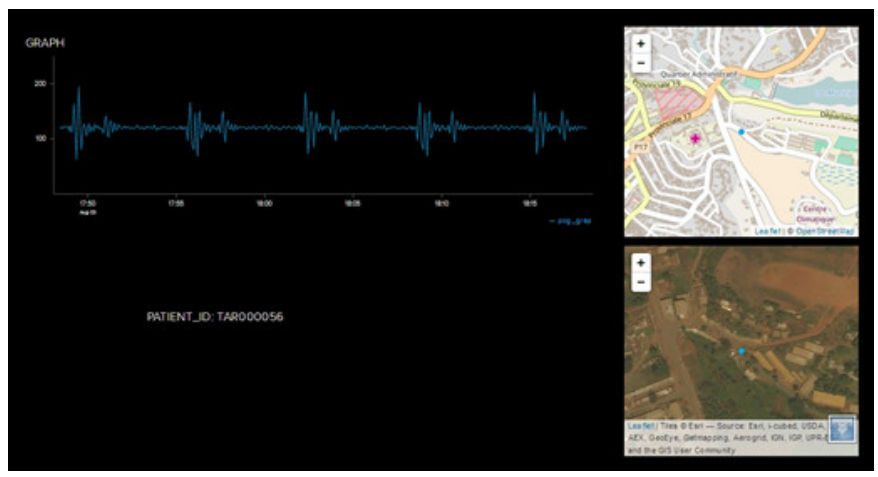

Figure 6: Example of geolocation and visualization of the mechanical activity of the core by MQTT cloud

\section{Conclusion}

We put in place a prototype for monitoring the mechanical activity of the heart based on a state-of-theart technique called the MQTT. The architecture of the surveillance prototype was presented. Typical networks for detecting the mechanical activity of the heart were introduced and compared. With the portable monitoring device containing a high-sensitivity microphone, real-time signals can be collected with satisfactory accuracy. The data collected was transmitted to the MQTT Cloud using Wi-Fi, which supports high data transfer rates and large coverage areas. MQTT Cloud is responsible for storing data and permitting doctors to view the signal. MQTT is also responsible for carrying out a geolocalization and to display it on the doctor's interface. Such a prototype can be used by hospitals and even by individuals due the fact that it is very potable and less costly. 


\section{REFERENCES}

[1] Lemondeinformatique., http://www.lemondeinformatique.fr/actualites/lire-mqtt-futur-protocole-de-linternet-des-objets-53422.html, 2017.

[2] Islam, S.M.R., et al., Internet of Things for health care: a comprehensive survey. IEEE Access, 2015. 3.

[3] Lars-Jochen, T., G. Colicchia and R. Girwidz, Phonocardiography with a smartphone. Phys. Educ. 2017. 52.

[4] Abdel-Motaleb, I.M., and V. K. Sambaraju, Development of a Wireless Cardiogram System for Acute and Long-term Healthcare Monitoring. EIT Conf, 2012.

[5] Mbiadoun, T., M. kom and P. N.Eloundou, Universal Module of Acquisition and Transmission of Electrophysiological Signal. IJIRSET, 2014. 3: p. 13767- 13776

[6] Jusak and I. Puspasari, Wireless tele-auscultation for phonocardiograph signal recording through the zigbee networks, IEEE Asia Pacific Conference on Wireless and Mobile (APWiMob), 2015.

[7] Sa-ngasoongsong, A., et al., A low-cost, portable, high-throughput wireless sensor system for phonocardiography applications. Journal of Sensors, 2012. 12: p. 10851-10870.

[8] Tchamda, A. R., et al., Low-Cost Phonocardiogram Board with Graphic LCD, Solar Panel and Embedded Heart Sound Analyser, IJIRSET, 2015. 4: p. 18793- 18799.

[9] Junaid M., et al., Internet of Things: Remote Patient Monitoring Using Web Services and Cloud Computing, Cyber-Physical-Social Computing, 2014.

[10] Zhao, Z, and Shengkang He, A Heart Sound Transmission and Reception System Based on NFC and Bluetooth, IFMBE Proceedings, 2014. 42: p. 187- 190.

[11] Chien, J. C, and C-C Tai; A New Wireless-Type Physiological Signal Measuring System Using a PDA and the Bluetooth Technology, Proceedings of the 2006 IEEE International Conference on Industrial Technology, 2006: p. 3026-3031.

[12] Amiri, A. M., K. Mankodiya, and G. Armano, PhonoSys: Mobile Phonocardiography Diagnostic System for Newborns, ICST , 2015.

[13] Hassanalieragh, M., et al., Health Monitoring and Management Using Internet-of-Things (IOT) Sensing with Cloud-based Processing: Opportunities and Challenges, IEEE computer society, 2015.

[14] Uttam U. D., and M. A. Kulkarni, IOT based Real Time ECG Monitoring System using Cypress WICED, ijareeie, 2017. 6(2).

[15] Savita, F., and S. Vashist, Design and Implementation of Wireless Body Area Network using Physiological Parameters, JOURNAL OF BIOMEDICAL ENGINEERING AND MEDICAL IMAGING, 2017. 4(1): p. 13-20.

[16] Van Rhijn, A., Integrated Circuits for High Performance Electret Microphones, Audio Engineering Society AES-E-Library the $114^{\text {th }}$ conference, 2003: p. 5719. 